\title{
Recognizing Human Activities from Multi-Modal Sensors
}

\author{
Shu Chen, Yan Huang \\ Department of Computer Science \& Engineering \\ University of North Texas \\ Denton, TX 76207, USA \\ Email: shuchen,huangyan@unt.edu
}

\begin{abstract}
This paper describes a method of detecting and monitoring human activities which are extremely useful for understanding human behaviors and recognizing human interactions in a social network. By taking advantage of current wireless sensor network technologies, physical activities can be recognized through classifying multi-modal sensors data. The result shows that high recognition accuracy on a dataset of 6 daily activities of one carrier can be achieved by using suitable classifiers.
\end{abstract}

\section{INTRODUCTION}

Detecting and monitoring human activities are extremely useful for understanding human behaviors and recognizing human interactions in a social network. There are many applications that can benefit from human activities recognition or detection. For example, patient monitoring is useful to inform nurses of patients' physical and mentor conditions in hospitals and senior houses. Current methods for tracking activities are time and resource consuming, relying on either paid observers or self-reports. Because of the tedious and intrusive nature, traditional methods of detecting and monitoring human activities are difficult and expensive. With the dramatic increase of the computation power of small devices and the emergence of new infrastructures such as wireless sensor networks, automatic human activity detecting becomes practical. An interesting fact about human activity detection is that environmental contextual information as well as social context gathered from wireless sensor devices can be used to detect social activities such as group meeting. The activity detection can be extended to prediction that can be used to practical applications such as avoiding calling-in during a group meeting. There were many studies on recognizing human activities using various sensory resources. Sensors such as cameras [1] or GPS have been used to detect a user's movement. Other researchers have used accelerometers [2] and magnetometers [3] as the main sensors to recognize activities such as walking, cycling, and writing.

In this paper, we describe a method that uses multi-modal sensors to help the recognition of human activities. Our system is based on Iris wireless sensor motes. The sensors attached to the motes collect information that includes temperature, acceleration, light intensity, sound, and magnetic field measurement. Together with external GPS and time information, we can obtain a reasonable set of contextual information about the carrier. The goal is to devise algorithms that can convert the raw and discrete multi-modal sensor readings to labeled human activities with semantic meaning. We experimentally demonstrated that using Iris sensor motes, a set of daily human activities can be successfully detected. We evaluated four types of classifiers and compared their performances. The dataset we collected and used in our experiments can also be accessed online.

\section{RELATED WORK}

Automatic human activities recognition is important for explaining human behaviors and understanding people's interactions and social roles in a social network. Tanzeem et al. [4] introduce some current approaches in activity recognition that use a variety of different sensors to collect data about users' activities. Further deployments and experiments are not provided in their paper. Joshua et al. [5] use RFID-based techniques to detect human-activity. Chen, D. [6] presents a study on the feasibility of detecting social interaction using sensors in skilled nursing facility. The work focuses on building statistical models from human labeled audio and visual data and simulated the algorithmic sensors. T. Nicolai et al. [7] use wireless devices to collect and analyze data in social context. Only Bluetooth detections are included in the study. Our approach extends current works of human activity recognition by using new wireless sensors with multiple modals. Our preliminary experiments show the feasibility of our approach and the results also indicate multi-modal sensors can improve the recognition accuracy of the classifiers.

\section{Method OvervieW}

Fig 1 gives an overview of our approach. The main idea is to use attached wireless sensor motes to collect raw data. Raw data integrated with manually recorded human activity labels are used as both training data to train activity models and testing data to test the accuracy of the methods, after preprocessing to eliminate noises and errors. Future data will be automatically labeled to certain physical daily activities using the models built. The labeled results can be used to summarize historical activities or predict future activities.

\section{DATA COLLECTION AND PREPROCESSING}

Personal daily activities are the focus of our research. We use Iris motes with MTS310 environmental sensorboard to record contextual data. Fig 2 and Fig 3 show the closure and 


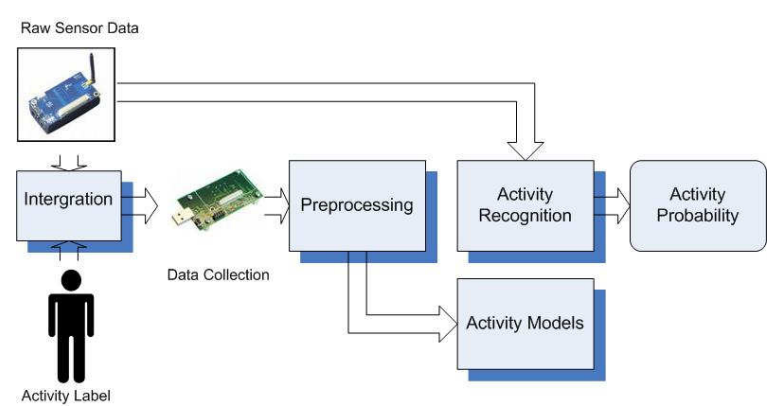

Fig. 1. Overview

Iris mote with MTS310. And a closure is attached to the waist of carriers. Carriers need to label daily activities with start time,finish time, and activity type as training data. Time series text data will be collected through one basestation (another Iris mote) to the data collection server.

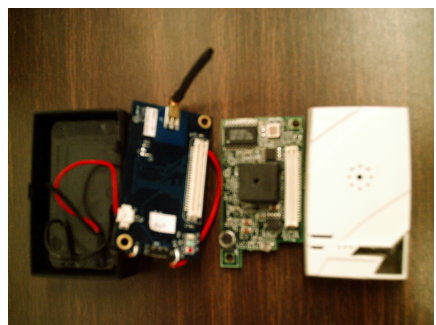

Fig. 2. Iris Mote with MTS310

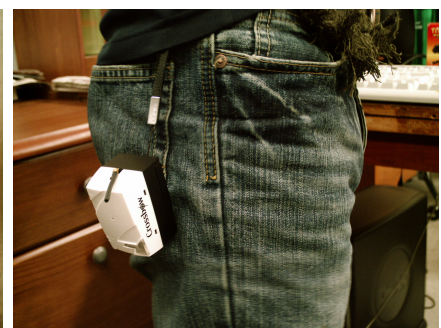

Fig. 3. Carrier with Closure

\section{A. Preprocessing}

In order to eliminate possible errors and get meaningful measurements, preprocessing is executed before classification. First, ADC readings received from MTS310 need to be converted into possible engineering unit. Table I shows the formula for converting the sensor readings into engineering unit. To avoid possible reading errors, - 99 was marked as error reading if reading error happened and was eliminated during preprocessing. Due to the consecutive nature, a single reading which is significantly different than readings before/after it is determined as noise and eliminated during preprocessing.

\begin{tabular}{|c|c|}
\hline Data Type & Formula \\
\hline Vref & $1.223 \times 1024 \div($ Read $)$ \\
Accelerate & $1000 \times(1-(500-$ Read $) \div 50$ \\
Temp & $1 \div(0.00130705+0.000214381 \times(\ln (10000$ \\
& $\times(1023 \times($ Read $))) \div($ Read $))+0.000000093$ \\
& $\times(\ln (10000 \times(1023-($ Read $))$ \\
& $\div($ Read $\left.))^{3}\right)-273.15$ \\
\hline
\end{tabular}

TABLE I

DATA CONVERTING

\section{B. Data Format}

The data formats and ranges are listed in Table II. Fig 4 gives a time series data example which records the data variation of one particular walking activity.

\section{Data Size and Activity Labels}

A list of six daily human activities (walking, running, driving, lecturing, meeting, and writing) are studied and collected

\begin{tabular}{|c|c|c|}
\hline Data Type & Format & Range \\
\hline Recording period & time & $5 \mathrm{~min}$ \\
Sensor ID & nx-uint8-t & $01-100$ \\
Location & $\mathrm{X}, \mathrm{Y}$ coordinate from GPS & \\
Temperature & nx-uint16-t & $-10-50$ Celsius \\
Accelerate & nx-uint16-t & $0-3000 \mathrm{millG}$ \\
Light & nx-uint16-t & $0-1024 \mathrm{ADC}$ \\
Micphone & nx-uint16-t & $0-1500 \mathrm{ADC}$ \\
Magnetic & nx-uint16-t & $0-1000 \mathrm{ADC}$ \\
Sequential number & nx-uint16-t & $0-60$ \\
\hline
\end{tabular}

TABLE II

DATA FORMAT

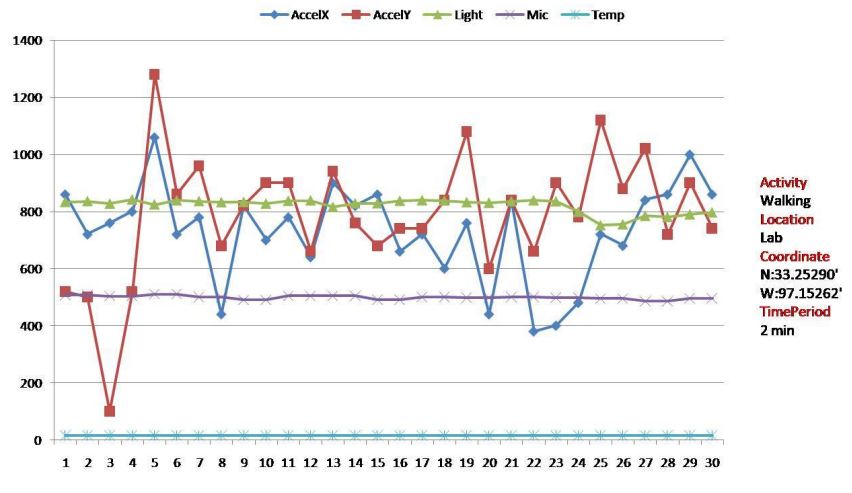

Fig. 4. Sample Data

in our dataset. The total numbers of time series data for each activity are different ( 2 for meeting; 2 for running; 4 for walking; 8 for driving; 12 for lecturing; 6 for writing). Each time series contains about 30 instances. Activity labels are chosen to reflect the content of the actions but do not specify the style. For instance, walking could be parameterized by walking speed and quantized into slow and fast. After preprocessing and eliminating possible errors, total 730 instances and 10 attributes are used in our experiments. The dataset is available to be download at:

http://groucho.csci.unt.edu/activity-recognition

\section{EXPERIMENTAL RESULT}

In this section, we use 4 different kinds of classification methods to recognize the activities. The differences through the result and some important observations are also analyzed and discussed in this section.

\section{A. Comparison}

We choose Random Tree (random classifier), KStar (nearest neighbor), J48 (decision tree), and Naive Bayes as classifiers to recognize the activities based on 7 features of our dataset (MagX, MagY, Micphone, Light, AccelX, AccelY, Temperature). $80 \%$ data of each activity are used for training and the rest 20\% are used for testing. The comparison result is showed in Table III.

\section{B. Analysis}

The result shows that all classifiers can achieve recognition accuracy above $92 \%$ for the dataset of a single carrier. Classification accuracy rates are between [95\%, 99\%] for walking, driving and lecturing which is extremely high comparing with the rest of activities (not shown in the table). Table IV shows the detailed classification results of J48 as an example which 


\begin{tabular}{|c|c|c|c|c|}
\hline Classifier & NaiveBayes & J48 & Random Tree & KStar \\
\hline Correctly Classified Instances & $92.4658 \%$ & $99.3151 \%$ & $98.6301 \%$ & $97.9452 \%$ \\
Incorrectly Classified Instances & $7.5342 \%$ & $0.6849 \%$ & $1.3699 \%$ & $2.0548 \%$ \\
Kappa statistic & 0.9045 & 0.9913 & 0.9826 & 0.974 \\
Mean absolute error & 0.0283 & 0.0015 & 0.0038 & 0.0159 \\
Root mean squared error & 0.1406 & 0.0319 & 0.0574 & 0.0719 \\
Relative absolute error & $10.8551 \%$ & $0.5834 \%$ & $1.4586 \%$ & $6.103 \%$ \\
Root relative squared error & $38.5601 \%$ & $8.7385 \%$ & $15.7535 \%$ & $19.7173 \%$ \\
\hline
\end{tabular}

TABLE III

COMPARISON RESULT

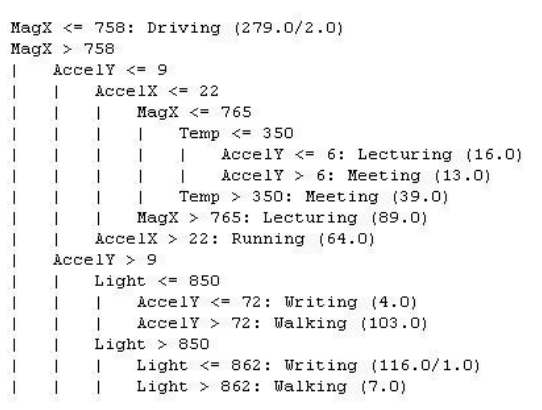

Fig. 5. J48 Pruned Tree

includes the True Positive (TP), False Positive (FP), Precision, Recall, and F-Measure. Lower recognition accuracies for other activities suggest that higher level analysis is required to improve classification of these activities. For example, temporal information in the form of duration, time, and day of activities could be used to detect activities. And the decision tree algorithm used in this work can recognize activities in high accuracy compared with other algorithms. One important observation is that not all the sensor readings are useful for determining the activity types. Fig 5 shows the J48 pruned tree. In this case, only MagX, AccelY, AccelX, Light, and Temperature are used to construct the decision tree. Another observation (observed from comparing the accuracy between using single sensor and multi-type sensors) shows that by using multi-modal sensors the accuracy significantly increases. The accuracy we get from training J48 using different single type of sensor is $33.5616 \%$ for single micphone data, 84.9315 $\%$ for single light, and $96.5753 \%$ for single acceleration respectively. Activities from different carriers will introduce more troubles for the classifier. The accuracy will drop to $63.4 \%$ when we use J48 to distinguish walking from the data of 4 carriers in our preliminary experiments.

\begin{tabular}{|c|c|c|c|c|c|}
\hline Class & TP Rate & FP Rate & Precision & Recall & F-Measure \\
\hline Walking & 0.968 & 0 & 1 & 0.968 & 0.984 \\
Driving & 0.98 & 0 & 1 & 0.98 & 0.99 \\
Lecturing & 0.95 & 0 & 1 & 0.95 & 0.974 \\
Running & 1 & 0.007 & 0.909 & 1 & 0.952 \\
Meeting & 1 & 0.008 & 0.933 & 1 & 0.966 \\
Writing & 1 & 0.008 & 0.957 & 1 & 0.978 \\
\hline
\end{tabular}

TABLE IV

J48 ACCURACY BY CLASS

\section{CONCLusion AND Future Work}

Result shows all of 4 classifiers can reach high recognition accuracy rate and by using decision tree classifiers recognition accuracy can reach $99 \%$ on a variety of 6 daily activities.
These results are competitive with prior activity recognition results that use only acceleration data. Furthermore, this work shows multi-modal sensor readings can be used to recognize a variety of human activities for human behavior detection. Further research will be needed to show how to use temporal data to determine the sequential patterns and predict future activities. How to improve accuracy by studying the temporal patterns of the acceleration and micphone is another issue to be addressed in future. And combining models built from multiple carriers to build a robust model for new carriers (so that she/he does not need to label and training her/his model) is another interesting topic.

\section{REFERENCES}

[1] L. Xie, S. fu Chang, A. Divakaran, and H. Sun, "Unsupervised mining of statistical temporal structures," in Video Mining, Azreil Rosenfeld, David Doermann, Daniel Dementhon Eds. Kluwer Academic Publishers, 2003.

[2] N. Ravi, N. Dandekar, P. Mysore, and M. L. Littman, "Activity recognition from accelerometer data," American Association for Artificial Intelligence, 2005. [Online]. Available: http://paul.rutgers.edu/ nravi/ accelerometer.pdf

[3] E. R. Bachmann, X. Yun, and R. B. Mcghee, "Sourceless tracking of human posture using small inertial/magnetic sensors," in Computational Intelligence in Robotics and Automation, 2003. Proceedings. 2003 IEEE International Symposium on, vol. 2, 2003, pp. 822-829 vol.2. [Online]. Available: http://ieeexplore.ieee.org/xpls/abs_all.jsp?arnumber=1222286

[4] T. Choudhury, M. Philipose, D. Wyatt, and J. Lester, "Towards activity databases: Using sensors and statistical models to summarize people's lives," IEEE Data Eng. Bull., vol. 29, no. 1, pp. 49-58, 2006.

[5] J. R. Smith, K. P. Fishkin, B. Jiang, A. Mamishev, M. Philipose, A. D. Rea, S. Roy, and K. Sundara-Rajan, "Rfid-based techniques for humanactivity detection," Commun. ACM, vol. 48, no. 9, pp. 39-44, September 2005. [Online]. Available: http://dx.doi.org/10.1145/1081992.1082018

[6] D. Chen, J. Yang, R. Malkin, and H. D. Wactlar, "Detecting social interactions of the elderly in a nursing home environment," ACM Trans. Multimedia Comput. Commun. Appl., vol. 3, no. 1, p. 6, 2007.

[7] T. Nicolai, E. Yoneki, N. Behrens, and H. Kenn, "Exploring social context with the wireless rope," in On the Move to Meaningful Internet Systems 2006: OTM 2006 Workshops, 2006, pp. 874-883. [Online]. Available: http://dx.doi.org/10.1007/11915034_112 